\title{
Beam Pulse Length and Guide Field Strength Parameter Study for the Autoresonant
} Acceleration Proof-of-Principle Experiment

\section{MASTER}


LA-7758-MS

Informal Report

UC-28

Issued: April 1979

\title{
Beam Pulse Length and Gulde Field Strength Parameter Study for the Autoresonant Acceleration Proof-of-Principle Experiment
}

\author{
Brendan B. Godfrey \\ Rickey J. Faehl
}

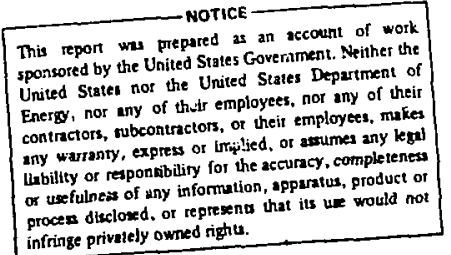


REAM PULSE LENGTH AND GUIDE FIELD STRENGTH PARAMETER STUDY FOR THE AUTORESONANT ACCELERATION PROOF-OF-PRINCIPLE EXPERIMENT

by

Brendan B. Godfrey and Rickey J. Faehi

\begin{abstract}
Generator and diate problems have reduced electron beam parameters for the proof-of-principle autoresonant collective ion acceleration experiment from $3 \mathrm{MeV}, 30 \mathrm{kA}$, and $200 \mathrm{~ns}$ to $2.25 \mathrm{MeV}, 15 \mathrm{kA}$, and $100 \mathrm{~ns}$. This reduction limits acceleration of hydrogen ions to about $4 \mathrm{MeV}$, if present experimental plans are followed. The output ion energy can be increased to a more acceptable level by reducing the electron beam radius at the diode and by accelerating ions in a weaker guide magnetic field. Analysis further suggests that preacceleration of ions is necessary for efficient trapping in the slow cyclotron waves. The maximum ion current is of order $15 \mathrm{~A}$.
\end{abstract}

I. INTRODUCTION

Hardware problems continue to plague the Austin Research Associates, Inc. (ARA) autoresonant collective ion acceleration proof-of-principle experiment. It now appears that the useful electron beam pulse for the experiment will be $100 \mathrm{~ns}$ long (flat top portion) at a current of $15 \mathrm{kA}$ and an energy of $2.25 \mathrm{MeV} .^{1}$ This represents a substantial reduction from originally projected values of $200 \mathrm{~ns}, 30 \mathrm{kA}$, and $3 \mathrm{MeV}^{2}$ In a recent report we analyzed the effects of the reduction in beam current and energy, finding that acceleration lengths were increased by a factor of approximately $2.5 .^{3}$ However, no account was taken of the change in pulse length. We were unaware of the change at that time. 
Pulse length is, however, very important. We find here that the new electron beam parameters limit hydrogen ion energy to $4 \mathrm{MeV}$, if the original experimental plans are otherwise followed. Operating at weaker guide-field strengths increases ion energy to $7 \mathrm{MeV}$. As noted in Ref. 3, decreasing the beam radius further increases maximum ion energy. Since $4 \mathrm{MeV}$ obviously is unacceptable even for a proof-of-principle experiment, redesign of the experiment is called for.

This report also addresses ion loading in the slow cyclotron waves. For any reasonable combination of initial wave phase velocity and well depth, trapping of stationary ions does not appear possible. Some sort of ion injector or preacceleration mechanism is required to bring the initial ion velocity up to that of the cyclotron waves. The corresponding ion energy is of order $1.25 \mathrm{MeV}$ according to current plans.

Initial loading of the cyclotron waves with ions places limits on the ion currents. For the present electron beam parameters, maximum ion current is of order $15 \mathrm{~A}$. This estimate is very crude.

Section IJ discusses the effects on ion acceleration of electron beam Fulse length, providing numerical data for a number of specific cases. Both aspects of the ion-loading process, the need for preacceleration and the ion current limit, are treated in Sec. III. Section IV summarizes our findings.

\section{ION ACCELERATION}

We consider first ion acceleration by an electron beam with original experimental design parameters, $30 \mathrm{kA}, 3 \mathrm{MeV}$, and $200 \mathrm{~ns}$. An ion energy of $30 \mathrm{MeV}$ is desired. As before, the variation of wave properties and the corresponding increase in ion enexgy are determined from conservation of wave energy flux and from the ion equations of motion. The principle point of our comp:atations here is to establish the time required for ion acceleration. The elapsed time is related to quantities already known simply by

$$
\mathrm{t}=\int \mathrm{dz} /
$$

Figure 1 shows the variation with time of the wave phase and group velocities, the axial electric field as felt by a moving ion, the relative modulation locally of the beam radius, the ion energy, the wave potential well depth, and the ion position. Potential well depth and ion energy are in MeV, the electric 
field in MV/cm, the axial distance in $\mathrm{cm}$, and the time in $\mathrm{ns}$. In this calculation the axial magnetic field decreases from 25.5 to $1.7 \mathrm{~kg}$. The wave frequency is chosen to be $286 \mathrm{MHz}$, corresponding to a phase velocit,y of $0.022 \mathrm{c}$ at maximum magnetic field strength, where ion loading occurs. The amplitude of the cyclotron wave at the entrance to the acceleration region is set to $\delta a=a / 2$. Larger values of the beam envelope modulation are impractical. From Fig. 1 we see that $165 \mathrm{~ns}$ is needed to achieve the desired ion energy of $30.0 \mathrm{MeV}$. The experimental design, in principle, can quite comfortably attain its goal. Incidentally, cutting the pulse length to $100 \mathrm{~ns}$ but leaving beam current and voltage unchanged gives an ion energy of $17.4 \mathrm{MeV}$, below design goals but probably still acceptable. Ion energy scales as

$$
\mathrm{U}_{i} \propto\left(\overline{\mathrm{E}}_{z} \tau\right)^{2}
$$

with $\overline{\mathrm{E}}_{z}$ the average axial electric field experienced by an ion. Since the axial electric field decreases slowly with decrease in the magnetic guide fields, $\bar{E}_{z}$ is about $25 \%$ larger for 100 ns of acceleration than for $165 \mathrm{~ns}$ of acceleration, partially offsetting the loss in ion erergy due to the shorter acceleration time alone.

Figure 2 gives results for currently available electron beam parameters, $15 \mathrm{kA}$ and $2.25 \mathrm{MeV}$. The wave frequency is increased to $350 \mathrm{MHz}$ in order to keep the wave phase velocity for ion loading unchanged. Otherwise, conditions are the same as for Fig. 1. With a full 200-ns pulse ion energy is 10.1 MeV. This value is nearly a factor of three below plans but is perhaps still tolerable. However, for a 1j0-ns pulse ion energy drops to $3.9 \mathrm{MeV}$, which is uniacceptable.

Apart from the obvious solution of increasing electron beam current and pulse length, two approaches to improving ion energy suggest themselves. As mentioned in the earlier report, reducing the electron beam radius by a factor of two fully compensates the loss of beam current. 3 The improvement results from decreasing the ratio of beam radius to cyclotron wavelength, leading to a much increased potential well depth for a given wave modulation amplitude. 4,5 Reducing the beam radius with the magnetic field profile held fixed necessitates redesign of the generator diode and of the beam drift tube. Although unappealing, this course of action is feasible experimentally. 
A third option is to reduce the maximum axial magnetic field and, thereby, to operate at longer cyclotron wavelengths. This method also decreases the ratio of beam radius to cyclotron wavelength, but is less successful than the proceding approach in that the increase in cyclotron wavelength tends to decrease the electric field for a given well depth, partially offsetting the gain in well depth. Figure 3, computed for a magnetic field ranging between 17.0 and $1.7 \mathrm{~kg}$, is a typical case. For a 200-ns pulse ions reach $14.0 \mathrm{MeV}$, while for a 100-ns pulse they reach $6.0 \mathrm{MeV}$. We have also examined the case of a 11.9- to 1.7-kg field profile. Further improvement is insignificant, with ion energy after $100 \mathrm{~ns}$ rising to $7.0 \mathrm{MeV}$. Therefore, adjustment of the guide field profile is by itself inadequate for bringing ion energy back to a reasonable value. Used in conjunction with the first two approaches, however, it seems quite fruitful. Experimental modifications needed are minor, and such related tasks as adiabatic compression actually are simplified.

The various cases analyzed are summarized in Table I. Listed are the electron energy in $\mathrm{MeV}$, the electron current in $\mathrm{kA}$, the wave frequency in $\mathrm{MHz}$, the pulse length in $\mathrm{ns}$, the axial magnetic field traversed in $\mathrm{kg}$, the corresponding acceleration length in $\mathrm{cm}$, the ion energy reached in $\mathrm{MeV}$, and the initial potential well depth in $\mathrm{kV}$. The potential well depth at maximum magnetic field strength is pertinent to ion trapping. It is discussed in Sec. III.

\section{TABLE I}

MAXIMUM ION ENERGY, ACCELERATION DISTANCE, AND INITIAL POTENTIAL

WELL DEPTH FOR VARIOUS COMBINATIONS OF ELECTRON ENERGY

AND CURRENT, PULSE LENGIH, AND MAGNETIC FIELD PROFILE. THE WAVE

FREQUENCY IS CHOSEN SO THAT THE INITIAL PHASE VELOCITY IS ROUGHLY $0.023 \mathrm{c}$.

\begin{tabular}{|c|c|c|c|c|c|c|c|}
\hline $\begin{array}{c}\mathrm{U} \\
(\mathrm{MeV})\end{array}$ & $\begin{array}{c}I_{e} \\
(k A)\end{array}$ & $\begin{array}{c}w_{0} \\
(\mathrm{MHz})\end{array}$ & $\begin{array}{c}\mathrm{B}_{0} \\
(\mathrm{~kg})\end{array}$ & $\begin{array}{c}\tau \\
\text { (ns) }\end{array}$ & $\begin{array}{c}Z \\
(\mathrm{~cm})\end{array}$ & $\begin{array}{c}\mathrm{U}_{\mathbf{i}} \\
(\mathrm{MeV})\end{array}$ & $\begin{array}{r}\phi_{t} \\
(\mathrm{kV})\end{array}$ \\
\hline 3.00 & 30 & 286 & $25.5-1.9$ & 165 & 814 & 30.0 & 25 \\
\hline 3.00 & 30 & 286 & $25.5-2.5$ & 100 & 361 & 17.4 & 25 \\
\hline 2.25 & 15 & 358 & $25.5-3.8$ & 200 & 509 & 10.1 & 4 \\
\hline 2.25 & 15 & 358 & $25.5-6.7$ & 100 & 175 & 3.9 & 4 \\
\hline 2.25 & 15 & 239 & $17.0-2.1$ & 200 & 669 & 14.0 & 14 \\
\hline 2.25 & 15 & 239 & $17.0-3.3$ & 100 & 215 & 6.0 & 14 \\
\hline 2.25 & 15 & 167 & $11.9-1.7$ & 146 & 419 & 10.5 & 30 \\
\hline 2.25 & 15 & 167 & $12.9-2.2$ & 100 & 246 & 7.0 & 30 \\
\hline
\end{tabular}


Before closing this section, it is important to note that the times cited are for acceleration only and do not include times for wave growth, ion loading, etc. Wave growth should consume no more than about 5 ns of the flat-top pulse. Ion loading may require 10 to $20 \mathrm{~ns}$, depending on details of the methods used.

\section{ION LOADING}

The last column of Table I lists for each case considered the cyclotron wave potential well depth available for phase-stable trapping of ions. Option two mentioned in the preceding section for improving ion energy, reducing the electron beam radius by a factor of two, results in an initial potential well depth computed in Ref. 3 to be $72 \mathrm{kV}$. For a fixed ratio of cyclotron frequency to plasma frequency, the potential well depth scales linearly with beam current. However, at short wavelengths the well depth varies inversely and very rapidly with the cyclotron frequency to plasma Erequency ratio. These dependencies are quite evident in Table $I$.

Experimental plans call for a cyclotron wave phase velocity in the range 0.020 to $0.025 \mathrm{c}$ at the point of ion loading. A hydrogen ion moving at these speeds has an energy of order $250 \mathrm{keV}$. It is clear, therefore, that stationary ions cannot be picked up by the cyclotron waves under the conditions considered so far. Two remedies are apparent. Either the wave velocity can be reduced or the ions can be preaccelerated before trapping.

Reducing the initial wave phase velocity while keeping the final velocity unchanged requires an increase in the axial variation of the guide magnetic field, because

$$
v_{i}^{\text {final }} / \mathrm{v}_{i}^{i n i t} \cong \mathrm{B}_{0}^{\max } / \mathrm{B}_{0}^{\min }
$$

Consider now the parameters of the last line in Table I. In order to trap scationary ions in a $30-\mathrm{kV}$ potential well, the initial wave velocity must be reduced by a factor of three, giving for the right side of Eq. (3) the value 16.2. Let us choose $B_{0}^{\mathrm{min}}=1.2 \mathrm{~kg}$, because for smaller fields no equilibrium exists. (In practice, operating so near the Brillouin limit probably is dangerous.) Then, $B_{0}^{\max }=19.4 \mathrm{~kg}$, and $\phi_{t}$ is reduced to roughly $11 \mathrm{kV}$. So, trapping still isn't possible. Similar results are obtained for the other sets of 
parameters from Table I. It appears that reduction of initial wave phase velocity is not, in itself, sufficient for effective ion loading.

Preacceleration of ions, on the other hand is effective and should not prove difficult. A $250-\mathrm{kV}$ ion gun is an obvious source of preacceleration. It may even be possible to use the DC fields of the electron beam itself, say by tapering the drift tube in the ion-loading region. 6

Let us now estimate the maximum ion current which can be accelerated. Suppose that $1 / 3$ of the injected ions are trapped by cyclotron waves and carried (ff, and $2 / 3$ remain in the ion loading section. If the density of accumulating icns is not to exceed the electron beam density, then

$$
I_{i}<\frac{1}{2} \frac{v_{i}}{c} \frac{\tau_{\ell}}{\tau} I_{e},
$$

where $\tau_{\ell}$ is the time required for any given cyclotron wave to pass through the ion-loading region and $\tau$ is the electron beam pulse length. Assuming $\tau_{\ell} / \tau \sim 0.1$, we find for present experimental parameters that $I_{i}$ is limited to about $15 \mathrm{~A}$. If electron-ion or ion-ion two-stream instabilities in the loading section prove sexious, then $I_{i}$ may have to be reduced further. On the other hand, if the residual ions can be swept out of the loading region, Eq. (4) becomes irreilevant.

A second estimate can be obtained by demanding that the average ion density near the beginning of the acceleration region not seriously exceed the density needed for force-neutral equilibrium. This yields

$$
I_{i}<2 \gamma^{-2} \frac{v_{i}}{c} I_{e}
$$

or, again, about $15 \mathrm{~A}$. It is important to remember that these two estimates are quite crude and should be viewed as giving order-of-magnitude values only. lacidentally, if available energy were the only constraint, ion currents as large as several hundred amps could be expected.

IV. CONCLUSIONS

This report first analyzes the effect of reduced pulse. length on the autoresonant acceleration proof-of-principle experiment. We find that present 
experimental parameters, line 4 of Table I, yield very little ion acceleration. Ilowever, even if electron beam parameters cannot be improved significantly, much greater ion acceleration can be achieved by decreasing the electron beam radius throughout the system and by operating at lower magnetic field strengths. More specifically, one should, in order of decreasing effect, (1) raise $I_{e}$ to $30 \mathrm{kA}$, (2) reduce the beam radius by a factor of two, (3) increase $\tau$ to $200 \mathrm{~ns}$, and (4) reduce $\mathrm{B}_{0}^{\max }$ to about $15 \mathrm{~kg}$.

The report next addresses ion-loading problems. In view of the small potential wells available for ion trapping, some sort of ion preacceleration scheme seems necessary. Ion current is limited to of order 15 A by the constraint that the electron beam equilibrium be not too strongly distorted by the ions.

\section{ACKNOWLEDGMENTS}

We are endebted to $\mathrm{Dr}$. Barry Newberger and Dr. William Shanahan for their helpful comments.

This research was supported jointly by the U. S. Army Ballistic Missile Defense Advanced Technology Center and by the U. S. Department of Energy. Dr. Larry Havard is Program Manager for BMDATC.

\section{REFERENCES}

1. Proof-of-Principle Experiment Program Review, Austin, Texas (18 January 1979), unpublished.

2. W. E. Drummond, G. I. Bourianoff, D. F. Brower, D. E. Hasti, W. W. Rienstra, M. L. Sloan, J. R. Thompson, J. R. Uglum, and H. V. Wong, "Proof of Principle Experiment for the Auto-Resonant Accelerator," Ausin Research Associates report I-ARA-76-U-100 (1976).

3. B. B. Godfrey and R. J. Faehl, "Beam Voltage and Current Parameter Study for tha Autoresonant Acceleration Proof-of-Principle Experiment," Los Alamos Scientific Laboratory report LA-7568-MS (1978).

4. B. B. Godfrey and B. S. Newberger, "Wave Amplitude Variation and Energy Flow in Autoresonant Collective Ion Acceleration," J. Appl. Phys., to be published.

5. B. B. Godfrey, "Linear Theory of Radially Inhomogeneous Unneutralized Relativistic Electron Beams," IEEE Plas. Sci., to be published. 
6. W. E. Drummond, G. I. Bourianoff, E. P. Cornet, D. E. Hasti, W. W. Rienstra, M. L. Sloan, J. R. Thompson, J. R. Uglum, and H. V. Wong, "Conceptual Design of an Auto-Resonant Accelerator Experiment," Air Force Weapons Laboratory report AFWL-TR-76-152 (1976).
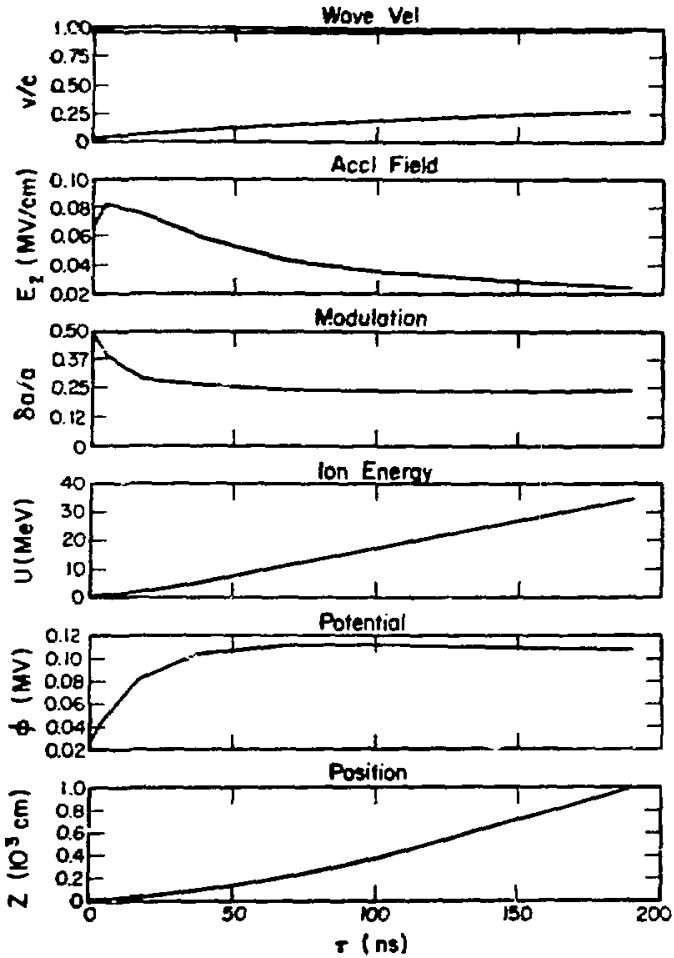

Fig. 1

Variation of cyclotron wave para meters, ion energy, and ion positica with elapsed time in the acceleratikn section of the proof-of-principlf autoresonant acceleration experiment for a $3-\mathrm{MeV}, 30-\mathrm{kA}$ electron beam. Wave frequency is $286 \mathrm{MHz}$. The magnetic guide field ranges from 25.5 to $1.7 \mathrm{~kg}$.
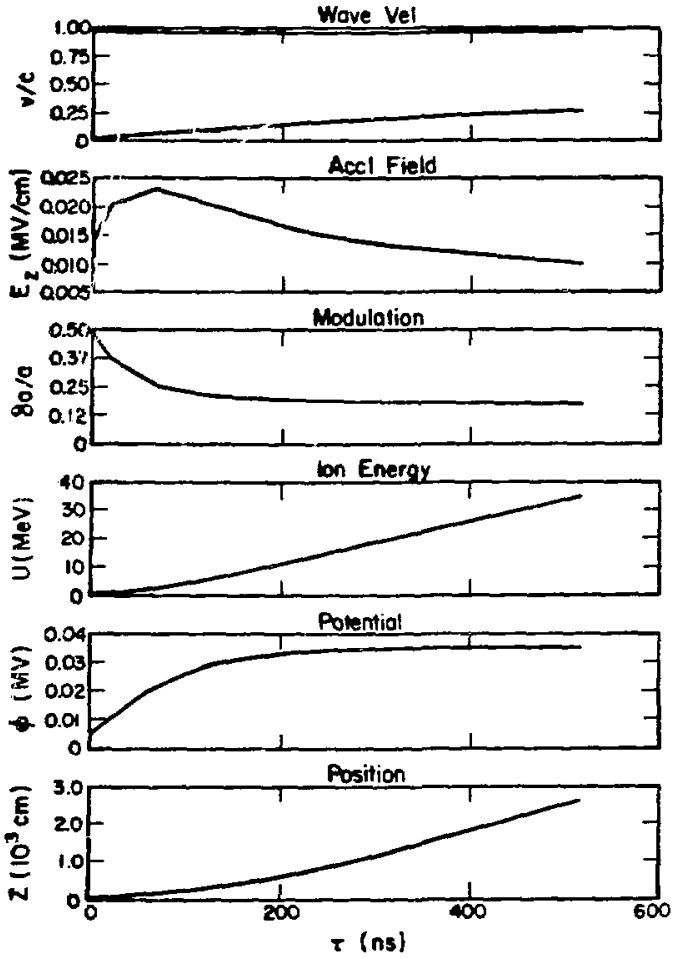

Fig, 2 .

Variation of cyclotron wave parameters, ion energy, and ion position with elapsed time in the acceleration section of the proof-of-principle autoresonant acceleration experiment fur a $2.25-\mathrm{MeV}, 15-\mathrm{kA}$ electron beam. Wave frequency is $358-\mathrm{MHz}$. The magnetic guide field ranges from 25.5 to $1.7 \mathrm{~kg}$. 

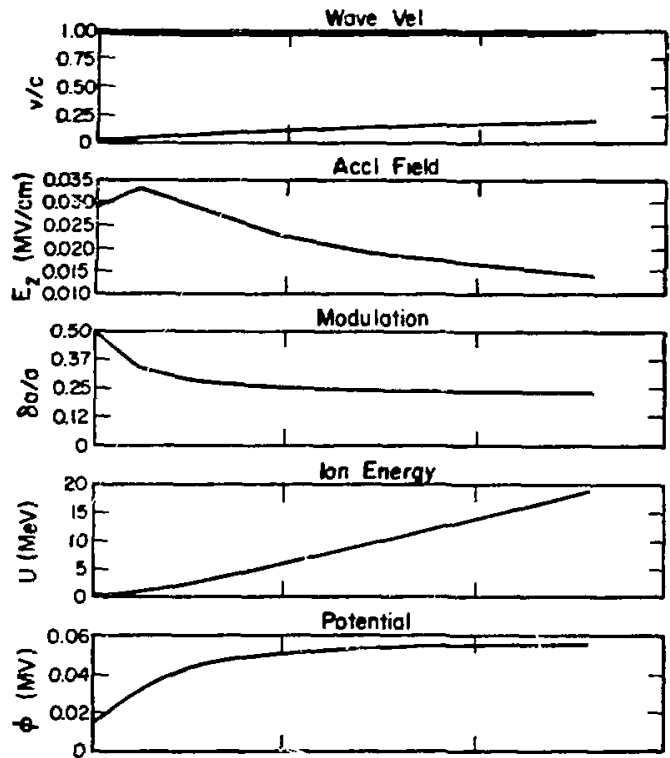

Fig. 3.

Variation of cyclotron wave parameters, ion energy, and ion position with elapsed time in the acceleration section of the proof-of-principle autoresonant acceleration experiment for a $2.25-\mathrm{MeV}, 15-\mathrm{kA}$ electron beam. Wave frequency is $239 \mathrm{MHz}$. The magnetic guide field ranges from 17.0 to $1.7 \mathrm{~kg}$.

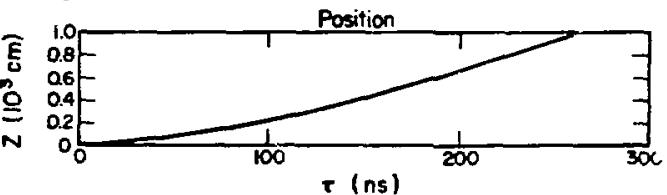

